\title{
Object Removal and Inpainting Technique by Lattice Boltzmann Method and Exemplar Method in Color Image
}

\author{
Liji R F, M Sasikumar, Sreejaya P, Kim J Seelan
}

\begin{abstract}
In this paper we use image inpainting technique in a color image. Inpainting is a technique in which, a missing area or pixels are replaced by adequate neighbouring pixels so that the resultant image obtained will look as no defect is there. In this paper we have removed an object from the color image and the vacant pixels are filled with the help of the surrounding neighbouring pixels. Two techniques are used here for removing and inpainting an object from the still color image, lattice boltzmann and exemplar method. These techniques were selected as Exemplar method of inpainting was used in many algorithms and have given a good result and lattice Boltzmann was usually used in experiments conducted in fluid dynamics. As motion of pixels can be compared to motion of fluids, lattice boltzmann method gives a better match for filling in the regions. Different variables for the inpainted image was calculated for both the methods. The signal to noise ratio and self-similarity index of the image is calculated for both inpainting techniques. According to the signal to noise ratio and the time taken for inpainting, it is found that lattice Boltzmann method of inpainting gives a better inpainted result..
\end{abstract}

Keywords : Diffusion, Exemplar method, Fluid dynamics, Image inpainting, Isophotone, Lattice Boltzmann method, Navier-stoke, Self-similarity index.

\section{INTRODUCTION}

Image inpainting is the technique in which, structural and textural corrections are made in a damaged image so that a better image is obtained [2]. Inpainting was used by art restorers initially. The manually done image restoration consume lot of human energy as well as time, therefore digital image inpainting techniques were introduced. With time of life, snapshots often get spoiled or scratched because of

Revised Manuscript Received on October 30, 2019.

* Correspondence Author

* Liji R. F*, Department of Electronics and Communication Engineering, John Cox Memorial CSI Institute of Technology, Thiruvananthapuram, Kerala, India, 9446892004, (lijirf1216@gmail.com)

Dr. Sreejaya P, Department of Electrical and Electronics Engineering, College of Engineering, Thiruvananthapuram, Kerala, India (sreejaya@ieee.org)

Dr. M. Sasikumar, Department of Electronics and Communication Engineering, Marian Engineering College, Thiruvananthapuram, Kerala, India. (drmsasikumar@yahoo.com)

Dr. Kim J Seelan, Department of Mechanical Engineering, John Cox Memorial CSI Institute of Technology, Thiruvananthapuram, Kerala, India, 9400368710, (kimseelan@gmail.com)

(C) The Authors. Published by Blue Eyes Intelligence Engineering and Sciences Publication (BEIESP). This is an open access article under the CC BY-NC-ND license (http://creativecommons.org/licenses/by-nc-nd/4.0/) humidity or other reasons. These deterioration can be reverted using inpainting. Now a days digital inpainting is widely used in various applications like remote sensing, for plucking of fruits or vegetables by robots, stereoscopic vision, etc.,. Inpainting can also be used in removing a particular object from the snapshot or for special effects. Removal of superimposed text like dates, subtitles, or publicity are the other adagial applications. Improved accuracy, high efficiency and quality. reduced time delay all made digital inpainting techniques an important one in today's fast world.

There are various inpainting algorithms under investigation. The inpainting techniques can be broadly classified as PDE based, Exemplar based, Hybrid based and Global based. Of which exemplar method is a renowned one [7]. In this paper we compare a lattice Boltzmann method (LBM) to an exemplar method. The LBM method used here choses a 8-neighbourhood pixel value to fill-in the missing region if the image $[10,11]$. And the image chosen is a painting here which is a complicated one. It is difficult task to inpaint a painting image. The exemplar method chosen works by diffusion of pixel values from known region to unknown region. The inpainting process will continue till all the missing pixels are replaced with the help of the surrounding area pixels. $\mathrm{S}$

\section{REVIEW OF LITERATURE}

Simultaneous structure and texture image inpainting was introduced in [1], the image feature was divided into two function. After applying inpainting to fill the structure as per the boundaries and capturing the noises, the regions were inpainted and finally added up. Bezier curves [3] introduced exemplar based inpainting inorder to reconstruct the missing information about the edge. Structure reconstruction was done in this and damaged areas were reconstructed using exemplar method. A fast texture matching algorithm based on multi-resolution and partition [4]. Here the image was made into blocks and sum of absolute difference was found out for each block and minimum value is eliminated. A variational model where it combined three basic techniques like PDE, neighboring pixel best match and copy paste [5]. The disadvantage of this method is that if the image does not have enough pixel to copy from, then the results obtained are poor after inpainting. 
A nonlocal Curvature -Driven Diffusion (CDD) model for image inpainting. In order to identify the lost pixel, pixels of similar structure rather than neighboring pixels are identified and filled to form inpainted image [6]. The drawback with this method is that it is effective only in images with same texture.

If this is implemented in an image with various texture, then the output is not clear. [7] gives the experimental analysis about successive elimination algorithm. The aesthetic method of photo retouching still image is given in [9]. The LBM methods used for smoothing and denoising are explained in $[10,11]$. As the computation is parallel in nature in LBM, the speed of processing is very high when compared to other methods explained in those papers. From these papers we can understand that LBM can be applied effectively in medical imaging for smoothening as well as denoising.

\section{LATTICE BOLTZMANN METHOD}

Lattice Boltzmann methods (LBMs) are used basically to simulate fluid flows. LBM originated from Lattice Gas Automata. They are now used in image processing techniques as they are good enough to solve complex boundaries. LBM has the capacity to remove any statistical noise in the image also. The particles live on the nodes of a discrete lattice, in lattice gases. In image processing the particles of gases can be related as pixels. The pixels move from one lattice node to next. On the way of propagation the pixels may collide with neighbouring pixels to attain a different value.

The general equation of LBM is DnQm Scheme. Here "Dn" stands for " $n$ dimensions" while "Qm" stands for "m neighbouring pixels. A two dimensional image with 8 neighbouring pixels are considered for this experiment. As 8neighbourhood of the image is taken a better inpainted image was received through our experiment. The values in $U$ are the neighbouring pixel values and g1 to g8 are the LBM parameters. $\mathrm{fj}$ is the LBM parameter. The algorithmics steps involved for finding LBM values are as follows :

1) Read the input image.

2) Find the size of input image.

3) Initialize the parameters Ux, Uy, Uxx, Uyy, Uxy, Unm, K.

4) Find Ux by taking the difference between the neighbouring rows.

5) Find Uy by taking the difference between the neighbouring columns.

6) Find Unm from Ux and Uy

7) Estimate value of Uxx and Uyy .

8) Find $\mathrm{K}$ from the estimated parameters;

9) Estimate LB parameters for g1,g2,g3,g4,g5, g6, g7, g8.

$$
\overrightarrow{f_{p}}=c \times\left\{\begin{array}{lr}
(0,0) & j=0 \\
(1,0),(0,1),(-1,0),(0,-1) & j=1,2,3,4 \\
(1,1),(-1,1),(-1,-1),(1,-1) & j=5,6,7,8
\end{array}\right.
$$

The Mask required for the Image inpainting can be prepared in many ways. The method we used is using paint. In paint, we can use eraser or any other tool to select the region to be inpainted. So the other part of that image will remains the same. Save that image and give that image as input to the coding algorithm in Matlab for the preparation of the mask. It consists of 0-255 grey level values so it provides value ' 1 ' for white portion and ' 0 ' for the rest. Thus the remaining portion changes to black. Thus we are getting the required mask. The algorithm to prepare the mask in is as follows:

1) Read the image.

2) Calculate the size of the image.

3) Select the area to be masked.

4) Find the area with grey value 1.

5) Formulate a for loop, until the selected area is replaced by grey value 255 .

6) Image with mask is obtained.

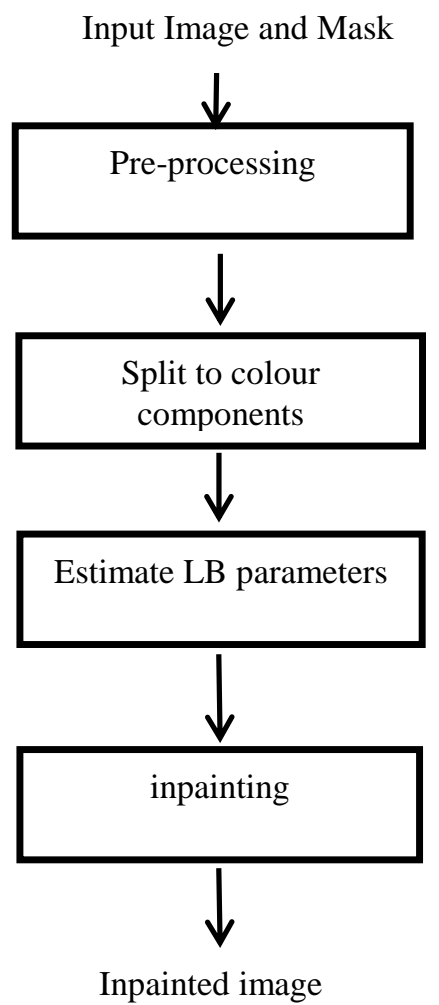

Fig. 1 Block diagram for Lattice Boltzmann method

\section{EXEMPLAR METHOD}

Exemplar-based inpainting can work in complex structures. It can also fill-in large gaps in images also. The pixel diffuse from the neighboring areas to the area to be inpainted. $[3,7,8]$ explain about exemplar based method using non-local means approach. The non - local approach gives a better inpainted output, as it take into account the details of the whole image. Following are the experimental steps for exemplar inpainting:

1. Input is given, which is the marked target region.

2. Confidence values are initialized.

3. Boundary of the target region is found out.

4. Patch priorities are found.

5. Patch with maximum priority is chosen and best exemplar is identified.

6. Update the missing region with exemplar and confidence value is updated.

7. Continue steps 2 to 6 until all the target region is filled.

8. Output obtained will be inpainted image. 


\section{EXPERIMENTAL RESULTS}

The experiment is carried out in Matlab 2016a in a personal computer with intel core i5 $7^{\text {th }}$ Gen. The image selected for inpainting is a picture which is has various structures and textures in it. Fig. 2(a-c) shows the original image, the mask created to remove the object and finally the inpainted image using lattice Boltzmann method. The object removed is the butterfly. Fig. 3(a-c) shows the images corresponding to exemplar method. The original image and the mask prepared are same for both the methods. Table 1 gives the different parameters of the image when experimented under lattice Boltzmann method and exemplar method. Various parameters like time taken for execution of the methods, the signal to noise ratio, the self-similarity index of the images with the original input image were calculated.

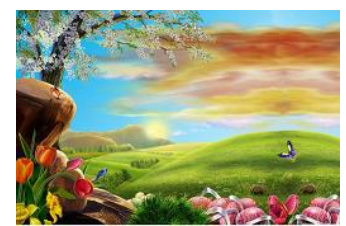

( a )

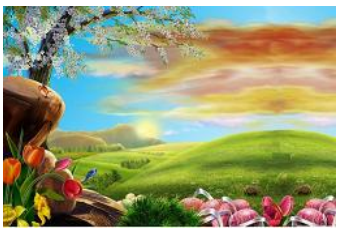

(C)

Fig. 2 Object Removal by LB Method (a) the input image. (b) the mask prepared to remove the object (c) the inpainted image after removal of object.

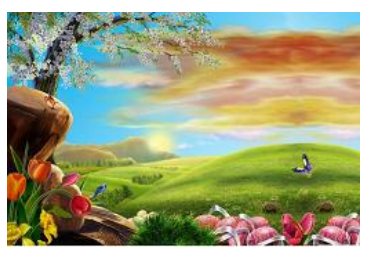

(a)

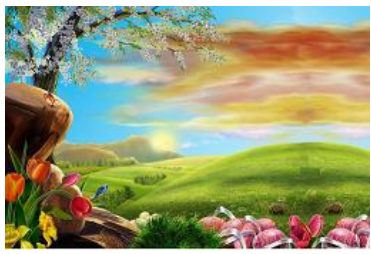

( c)

Fig.3. Object Removal by Exemplar Method (a) the original image (b)the mask prepared to remove the butterfly, which is the object and (c) the image after inpainting using exemplar method.

Table- I: Parameters of image for exemplar and lattice boltzmann method after inpainting was done.

\begin{tabular}{|l|l|l|}
\hline \multicolumn{1}{|c|}{ Items } & Exemplar method & $\begin{array}{c}\text { Lattice Boltzmann } \\
\text { Method (LBM) }\end{array}$ \\
\hline Size of the image & $256 \times 170$ pixels & 256 x 170 pixels \\
\hline
\end{tabular}

\begin{tabular}{|l|l|l|}
\hline Size of the mask & $256 \times 170$ pixels & $256 \times 170$ pixels \\
\hline Time & 20.68 seconds & 14.02 seconds \\
\hline $\begin{array}{l}\text { Signal to Noise Ratio } \\
\text { (SNR) }\end{array}$ & 42.3148 & 62.5248 \\
\hline $\begin{array}{l}\text { Structural similarity } \\
\text { index (SSIM) }\end{array}$ & 0.9769 & 0.9854 \\
\hline $\begin{array}{l}\text { Peak Signal to Noise } \\
\text { Ratio (PSNR) }\end{array}$ & $41.5 \mathrm{db}$ & $45.15 \mathrm{db}$ \\
\hline
\end{tabular}

\section{CONCLUSION \& FUTURE SCOPE}

Object removal and image inpainting is done using exemplar algorithm and lattice Boltzmann algorithm by using matlab and made a comparative study by verifying the parameters like execution Time, SNR, Size of object removed. The size of removed object by Exemplar algorithm can be more than in lattice Boltzmann algorithm. But as the size increases the executing time also increases. In our work we can use a size of image up to ( 1024×1024 pixels).

In general we concluded that, the LBM technique is more efficient in getting fast and good quality results than the Exemplar method. Because, the execution time for Exemplar method is more than LBM. LBM gives a better PSNR and SSIM also.

As a future scope these algorithms can be implemented in three dimensional images and for video inpainting and fuzzy logic can also be incorporated.

\section{REFERENCES}

1. Marcelo Bertalmio, Luminita Vese, Guillermo sapiro and Stanley Osher, "Simultaneous structure and texture Image Inpainting", IEEE Transactions on Image Processing, pp. 882 - 889, Vol.12, no. 8, Aug 2003.

2. M. Bertalmio, G. Saprio, V. Caselles, and C. Ballester, "Image Inpainting," Proceedings of the 27th annual conference on Computer graphics and interactive technique, 417-424, 2000.

3. Jason C Hung, Chun -Hong Hwang, Yi-Chun Liao, Nick C Tang, Ta-Jen Chen, "Exemplar-based Image Inpainting base on Structure Construction", Journal of Software, pp. 57 - 64, Vol.3, no.8, Nov. 2008

4. Shuhan Wei, Ranran Zhang, Pengyi Hao and Youdong Ding, "A Fast Texture Matching Algorithm Based on Multi-Resolution and Partition”, 5th IEEE Int. Conference on INC, IMS and IDC, pp.1240 - 1245, 2009.

5. Aurelie Bugeau, Marcelo Bertalmio, Vicent Caselles and Guillermo Sapiro, "A Comprehensive Framework for Image Inpainting", IEEE Transactions on image inpainting, Vol.19, no.10, pp.2634 - 2645, Oct. 2010.

6. Li Li and Han Yu, "Nonlocal Curvature-Driven Diffusion Model for Image Inpainting", 5th IEEE Int. Conf. on Information Assurance and Security, pp.513 - 516, 2009

7. Alexander Wong and Jeff Orchard, "A Non Local - Means Approach To Exemplar- Based Inpainting”, IEEE , pp 2600 - 2603, 2008.

8. Wang Shu-wen and Xa Yong-Sheng, "Improvement of Priority Computation in Exemplar - Based Image inpainting Based on D-S Evidence Theory and TV Model", IEEE, 2009

9. Hao Guo, "Aesthetic phot retouching with an image inpainting algorithm", IEEE, 2009.

10. Y chen, Z Z Yan, and Y H Qian, "Lattice Boltzmann method for Image Denoising", Tien Tzu Hsueh Pao/acta Electronica Sinica, Vol. 37, No.3, 2009, pp. $574-580$.

11. Y chen, Z Z Yan, and Y H Qian, "An Anisotropic Diffusion Model for Medical Image Smoothing by Using the Lattice Boltzmann Method", IFMBE Proceeding of ABCMBE2008, 2008, pp. 255 - 259.
Published By: Blue Eyes Intelligence Engineering \& Sciences Publication 


\section{AUTHORS PROFILE}

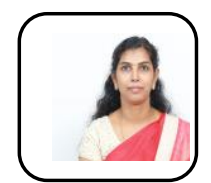

Liji R F was awarded Bachelor of Technology (2006) from University of Calicut , KMCT College of Engineering with First class in Electronics and Communication engineering and Master of Engineering (2008) was awarded to her from Anna University, Chennai, Sri Krishna College of engineering and Technology with First class in Communication Systems and now she pursues her $\mathrm{PhD}$ under University of Kerala. She works as Associate Professor and Head of the Department in Charge in John Cox Memorial CSI Institute of Technology, Trivandrum, India. She has 11 years' experience in teaching. She has guided a number of under graduate projects. She is a life member of ISTE.

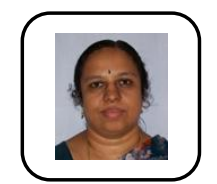

Sreejaya P was awarded Ph.D in Power systems from Kerala University. MTech from Kerala University and B.Tech from TKM college of Engineering under Kerala University in electrical and electronics Engineering department. She is the Professor and Head of the department at College of Engineering Trivandrum in the Department of Electrical \& Electronics Engineering Department. She has a number of publications in national and international journals. She has guided a number of PG and UG projects. She is a senior member of IEEE and a life member of ISTE. She has more than 28 years of teaching experience. She is the reviewer for IJEPES Elsevier Journal (Certified as outstanding reviewer) and for IEI series B Journal.

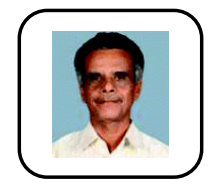

M. Sasikumar was awarded Ph.D (1985) in Circuits and Systems from Indian Institute of Technology, Madras. M.Tech (1976) Electrical Communication from Indian Institute of Science, Bangalore First Class with Distinction and B.Tech (1968) in Telecommunication from University of Kerala, College of Engineering, Trivandrum with First Class. Professional Body member of ISTE, CSI, IEI. He has guided $15 \mathrm{PhD}$ scholars. He has many publications in national and international journals. He was the principal of College of Engineering, Trivandrum (1997-1998). Presently he is the Head of the Department, Department of Electronics And Communication Engineering, Marian Engineering College, Trivandrum. He has a teaching experience of more than 50 years.

KIM J SEELAN was awarded Ph.D from NIU, Tamil Nadu in 2018. He is currently working as Associate Professor at John Cox Memorial CSI Institute of Technology, Trivandrum. He has guided many PG students. He has 10 year of teaching experience. He is a life member of ISTE. 